\title{
Uterus didelphys with multiple fibroids: A case report
}

\author{
Dr. Mohammed Khairy Ali, ${ }^{1}$ Dr. Ahmed Yehia Abdelbadee, ${ }^{1}$ Dr. Sherif Abd El- Karim \\ Mohammed Shazly, ${ }^{1}$ Dr. Ahmed Mohammed Abbas ${ }^{1}$
}

Keywords: uterus didelphys, fibroid, chocolate cyst, double uterus, mullerian duct

\begin{abstract}
Uterine anomalies are congenital malformations caused by fusion or resorption defects during embryogenesis. Uterus didelphys, or a double uterus, is a rare condition that occurs in female fetuses as they develop in the womb. Here we report a case of a 46 year old virginal female with uterus didelphys along with multiple fibroids of both uteri and a right ovarian cyst. The patient underwent a total abdominal hysterectomy with adnexa removal. The specimen showed leiomyoma of uterus with ovarian endometriosis.

${ }^{1}$ Women's Health Center, Assiut University,
Egypt
\end{abstract}

\section{Introduction}

Mullerian duct anomalies are congenital anomalies of the female genital tract resulting from non-development or nonfusion of the mullerian ducts or failed resorption of the uterine septum. ${ }^{1}$ Duplication of the uterus results from the lack of fusion of the paramesonephric ducts in a local area or throughout their normal line of fusion. ${ }^{2}$ In uterus didelphys, individual horns are fully developed and are normal in size, with two cervices inevitably present. Each individual horn is associated with one fallopian tube. Women with didelphic uteri may be asymptomatic and unaware of having a double uterus. They may present with complaints of dysmenorrhea and dyspareunia. ${ }^{3}$ The true prevalence of this anomaly is unknown as it may be discovered in the later part of a patient's life when presenting with infertility. The incidence of mullerian duct anomalies in the literature ranges from 0.5 to $5.0 \%$. Approximately $11 \%$ of uterine malformations are didelphic uteri. ${ }^{4}$

\section{Case history}

A 46 year old virginal female was admitted to the Women Health Hospital in Assiut University with a 4 month history of vaginal bleeding and a 2 week history of lower abdominal pain without dysmenorrhea. There was no past history of drug use or previous abdomino-pelvic surgery.

On general examination, the findings were unremarkable and the patient was otherwise healthy. On examination, there was an abdomino-pelvic mass equal to the size of a 34 week pregnant uterus by palpation. The mass was firm, irregular, was not tender, and had

Please cite this paper as: Ali MK, Abdelbadee AY, Shazly SA, Abbas MA. Uterus didelphys with multiple fibroids: A case report. Proc Obstet Gynecol. 2013;3(2):Article 3 [4 p.]. Available from: http://ir.uiowa.edu/pog/. Free full text article.

Corresponding author: Mohammed Khairy Ali, Women's Health Center, Assiut University, Egypt, m khairy2001@yahoo.com

This is an Open Access article distributed under the terms of the Creative Commons Attribution 3.0 Unported License (http://creativecommons.org/licenses/by/3.0), which permits unrestricted use, distribution, and reproduction in any medium, provided the original work is properly cited. 
limited mobility. Pelvic examination could not be performed as she was virginal. Hematological examination showed hemoglobin of $9.0 \mathrm{~g} / \mathrm{dL}$ and hematocrit of $26 \%$.

Abdominal ultrasound examination showed an enlarged uterus with a distorted endometrial lining and multiple subserous, interstitial and submucous fibroids. The adnexa could not be visualized. The patient was counseled for the possibility of hysterectomy, and informed consent for abdominal exploration was obtained.

An exploratory laparotomy through a midline vertical incision revealed multiple fibroids with different sizes arising from two separate uterine horns. Additionally, a cyst in the right ovary measuring $5 \mathrm{~cm} \times 5 \mathrm{~cm}$ was noted (Figure 1). A total hysterectomy with bilateral removal of adnexae was performed (Figure 2). Examination of the specimen after the procedure revealed the presence of two cervices to conclude the diagnosis of uterus didelphys. The intraoperative estimated blood loss was about 500 cc. However, based on preoperative hemoglobin levels and on the clinical observation of pallor, it was recommended to administer three units of blood intraoperatively. Postoperative follow-up was uneventful and the patient was discharged on the eighth post-operative day. Histopathological examination of the specimen confirmed the presence of multiple leiomyomata with a right endometriotic ovarian cyst.

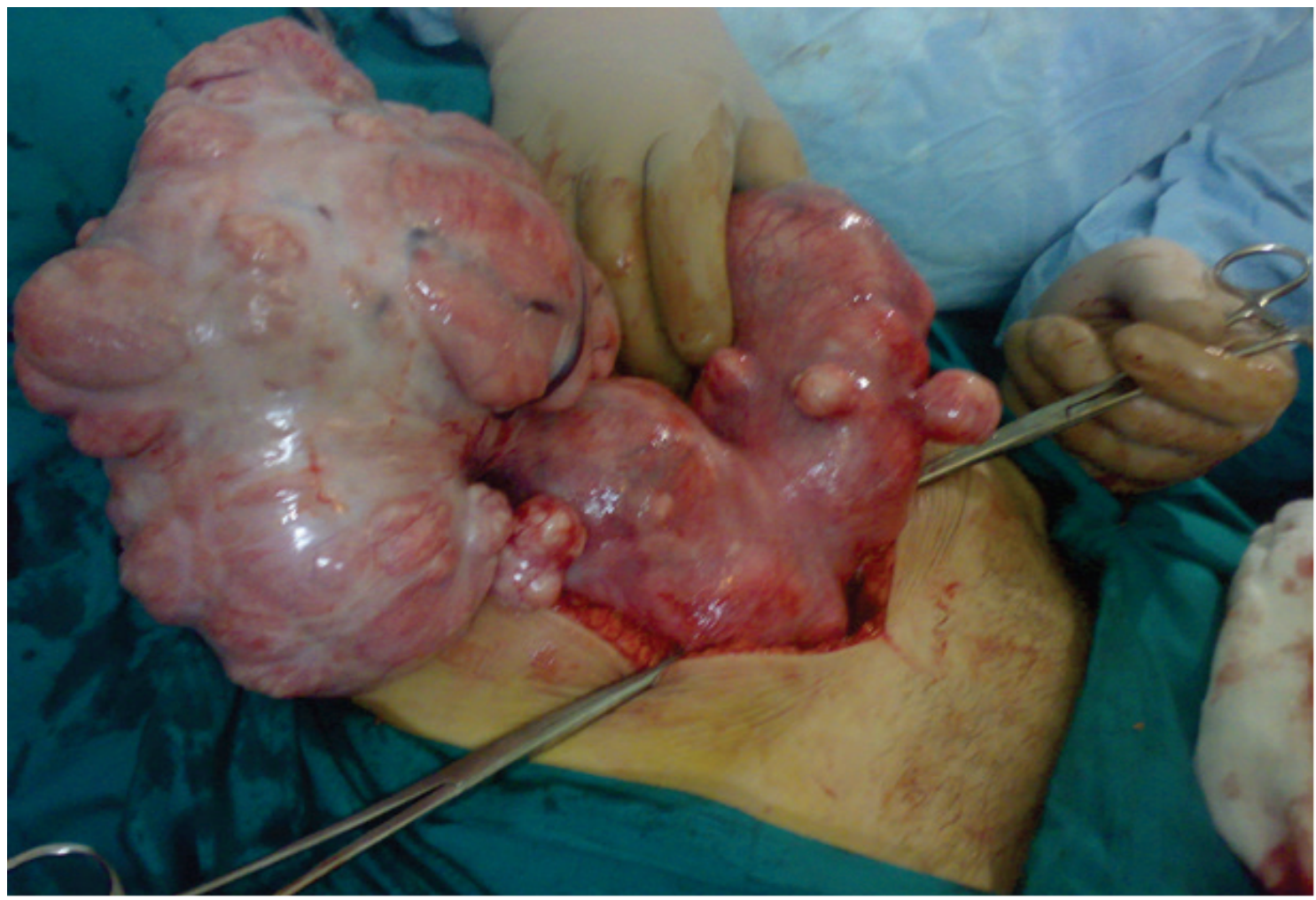

Figure 1: Intraoperative view shows uterus didelphys with multiple uterine fibroids 


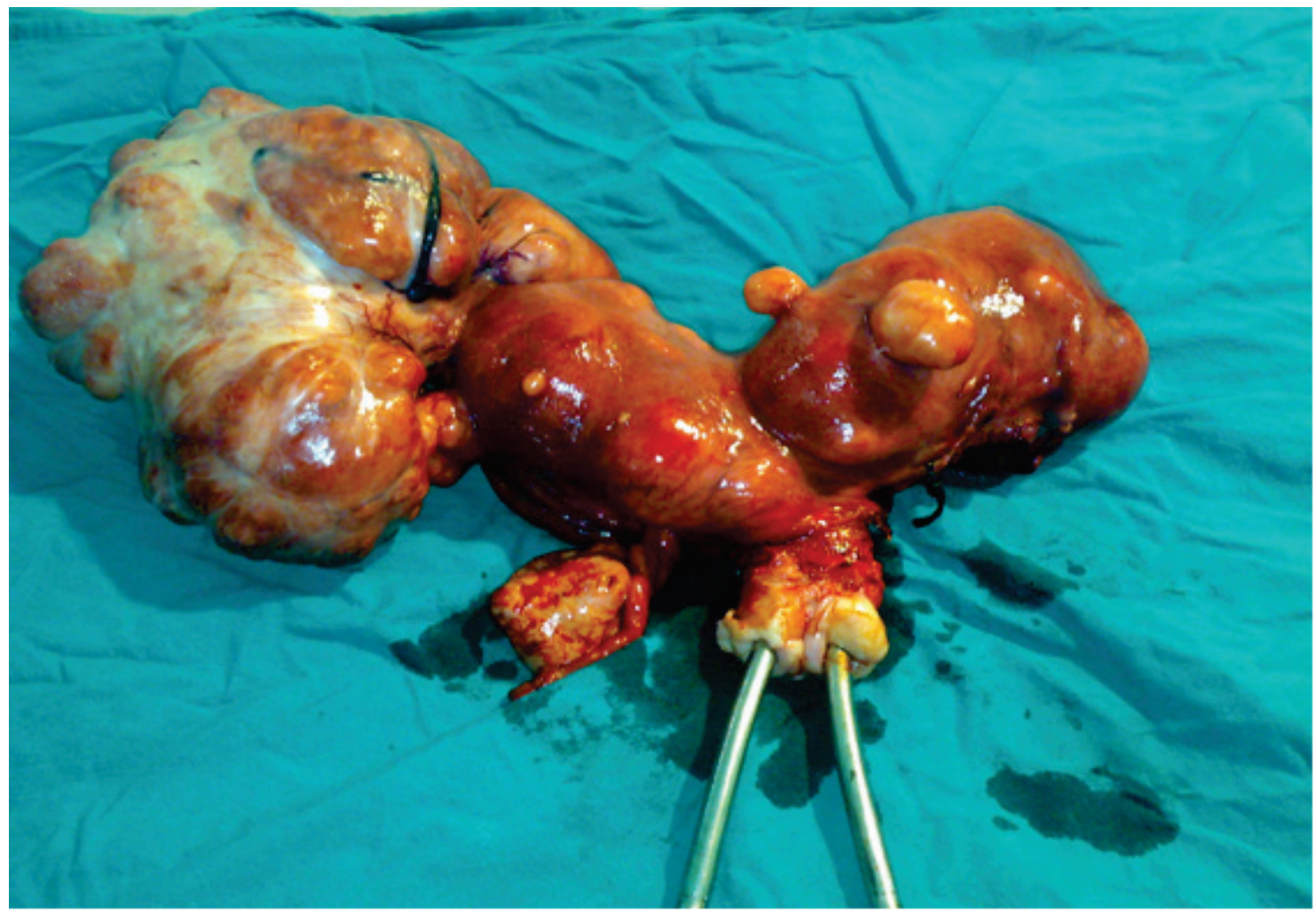

Figure 2: shows uterus didelphys after hysterectomy

\section{Discussion}

Uterus didelphys is a rare condition that develops in female fetuses. ${ }^{5}$ Normally, the two mullerian ducts are fused together to create a single uterus, but occasionally these two tubes fail to join. When this happens, two separate uteri form, with double cervices and potentially double vaginas as well. ${ }^{6}$ They are both fully functioning uteri, and there are rare cases of women with this condition that have given birth to twins who were each housed in a separate uterus. $^{7}$

The cause of this anomaly is not known and there are often no symptoms present, so many women are never diagnosed with uterus didelphys. Other women may have dysmenorrhea and some will have reproductive and pregnancy issues. ${ }^{8}$ If uterus didelphys is suspected, a pelvic exam can confirm the presence of a double vagina and/or double cervix. Diagnostic procedures should then be performed to evaluate the uterus, including a pelvic ultrasound, magnetic resonance imaging (MRI), and hysterosalpingography (HSG). ${ }^{9}$

Here we report a rare case of multiple fibroids within a uterus didelphys in addition to an endometriotic cyst. In our case, ultrasonography failed to diagnose uterus didelphys due to the presence of multiple fibroids with different sizes and at different sites. Also, diagnosis of uterus didelphys could not be performed by pelvic examination or HSG as the patient was virginal. Surgical management by total hysterectomy was the only method to determine the presence of two cervices and achieve 
the diagnosis of uterus didelphys.

There are very few similar cases reported in the literature. Generally, reported cases presented with infertility, and the presence of fibroid masses was thought to contribute in this problem. ${ }^{10}$ However, in this case, the patient was virginal. Accordingly, infertility can not be confirmed or excluded in this woman. The interesting point in this case is the unreported new presentation. The patient presented with dysmenorrhea that was found to be mostly related to ovarian endometrioma. This association is generally not reported and cannot be simply explained by mullerian duct abnormalities (unless associated with outflow obstruction). Furthermore, unlike other reported cases, fibroids were multiple. $^{11}$

\section{References}

1. Madureira AJ, Mariz CM, Bernardes JC, Ramos IM. Case 94: Uterus didelphys with obstructing hemivaginal septum and ipsilateral renal agenesis. Radiology. 2006 May;239(2):602-6. PubMed PMID: 16641359. http://dx.doi.org/10.1148/radiol.2392031 187

2. Nahum GG. Uterine anomalies. How common are they, and what is their distribution among subtypes? J Reprod Med. 1998 Oct;43(10):877-87. Review. PubMed PMID: 9800671.

3. Shulman LP. Müllerian anomalies. Clin Obstet Gynecol. 2008 Jun;51(2):214-22. doi: $10.1097 / G R F .0 b 013 e 31816 f e b a 0$. Review. PubMed PMID: 18463453.

4. Olpin JD, Heilbrun M. Imaging of Müllerian duct anomalies. Clin Obstet Gynecol. 2009 Mar;52(1):40-56. doi: 10.1097/GRF.0b013e3181958439.

Review. PubMed PMID: 19179860.
5. Lin PC. Reproductive outcomes in women with uterine anomalies. J Womens Health (Larchmt). 2004 JanFeb;13(1):33-9. Review. PubMed PMID: 15006276.

6. Verson RE, et al. Clinical manifestations and diagnosis of congenital anomalies of the uterus. http://www.uptodate.com/home/index.ht ml. Accessed July 27, 2010.

7. Iverson RE, et al. Surgical management of congenital uterine anomalies. http://www.uptodate.com/home/index.ht ml. Accessed July 27, 2010.

8. Saravelos $\mathrm{SH}$, Cocksedge KA, Li TC. Prevalence and diagnosis of congenital uterine anomalies in women with reproductive failure: a critical appraisal. Hum Reprod Update. 2008 SepOct;14(5):415-29. doi: 10.1093/humupd/dmn018. Epub 2008 Jun 6. Review. PubMed PMID: 18539641.

9. Li S, Qayyum A, Coakley FV, Hricak H. Association of renal agenesis and mullerian duct anomalies. J Comput Assist Tomogr. 2000 NovDec;24(6):829-34. PubMed PMID: 11105695 .

http://dx.doi.org/10.1097/00004728200011000-00001

10. Ayoola O, Alao O, Ibitoye B, Ma'aji S. Bicornuate Uterus With Associated Bilateral Tubal Blockage And Fibroid Tumors: A Rare Combination Causing Infertility. The Internet Journal of Radiology. 2007; 6:1. DOI: 10.5580/1bef

11. Ly JQ. Rare bicornuate uterus with fibroid tumors: hysterosalpingographyMR imaging correlation. AJR Am J Roentgenol. 2002 Aug;179(2):537-8. PubMed PMID: 12130475. 